\title{
Abundancia, reproducción y tallas del tiburón martillo Sphyrna lewini (Carcharhiniformes: Sphyrnidae) en la pesca artesanal de Golfo Dulce, Pacífico de Costa Rica
}

\author{
Ilena Zanella ${ }^{1} \&$ Andrés López-Garro ${ }^{1}$ \\ 1. Asociación Conservacionista Misión Tiburón, Playas del Coco, Guanacaste, Costa Rica; izanella@misiontiburon.org, \\ alopez@misiontiburon.org
}

Recibido 11-VIII-2014. Corregido 20-XI-2014. Aceptado 22-XII-2014.

\begin{abstract}
Abundance, reproduction and length of scalloped hammerhead shark Sphyrna lewini (Carcharhiniformes: Sphyrnidae) in the artisanal fishery in Golfo Dulce, Pacific of Costa Rica. Incidental catch of S. lewini by artisanal fishermen in Golfo Dulce, Costa Rica, suggest that this could be one of the coastal locations used by the species. This study aims to characterize the scalloped hammerhead shark population in Golfo Dulce, in order to best guide management actions for the conservation and sustainable use of the species. Fisheries-dependent biological data (size-TL, sex, reproductive status) were gathered for S. lewini between May 2010 and May 2011 in the communities of Puerto Jimenez and Pavones, Golfo Dulce during artisanal fishing trips. Catch per unit effort (CPUE) throughout the year was also estimated from catch data. We sampled a total of 315 sharks. Mean total length was estimated at $74.3 \pm 17.4 \mathrm{~cm}$. Both pups and juveniles were reported, which presented a male: female ratio of 1:1.2 ( $>>0.05)$. Total length and relative abundance of $S$. lewini had opposite tendencies, with the smallest TL $(64 \mathrm{~cm})$ recorded during July-August, when CPUE was highest. (0.0075). Temporary closures from June to August are therefore recommended at areas of highest reported catch (Pique Fijo, Los Bajos y La Ciénaga), in order to protect newly born S. lewini, and promote the sustainability of the species in the ETPS Closures should be complemented with a long-term study that can further clarify whether Golfo Dulce is a nursery area for S. lewini. Rev. Biol. Trop. 63 (Suppl. 1): 307-317. Epub 2015 April 01.
\end{abstract}

Key words: Scalloped hammerhead shark, Sphyrna lewini, nursery area, artisanal fishery, Gofo Dulce.

Antes de los años ochentas los tiburones representaban una captura incidental en la pesca de grandes peces pelágicos y debido a su bajo valor comercial, solían ser liberados (Barker \& Schluessel, 2005). Sin embargo, en las últimas décadas la pesca de tiburones ha experimentado un rápido crecimiento, fundamentalmente para suplir el mercado asiático de aleta de tiburón (Walker, 1998; Kitchell, Essington, Boggs, Schindler \& Walters, 2002; Abercrombie, Clarke \& Mahmood, 2005; Barker \& Schluessel, 2005). Debido a las características biológicas y la naturaleza migratoria de algunas especies, los tiburones se consideran especies muy vulnerables a la explotación pesquera, tanto así que su pesca dirigida ha ocasionado un drástico declive de sus poblaciones (Baum et al., 2003; Rowe \& Hutchings, 2003; Távarez, 2005; Myers, Baum, Shepherd, Powers \& Peterson, 2007). Como consecuencia, a nivel mundial se han realizado esfuerzos nacionales y regionales para promover su conservación. Por ejemplo, en el marco del Paisaje Marino del Pacífico Este Tropical (ETPS, por sus siglas en inglés) se han desarrollados proyectos para promover la conservación y manejo de los tiburones en Panamá, Costa Rica, Colombia y Ecuador (Hearn, Utreras \& Henderson, 2010). Una de las especies de interés para el ETPS es el tiburón matillo, Sphyrna lewini. Dicha especie se encuentra en la Lista Roja de la UICN (Unión Internacional para la Conservación de 
la Naturaleza) como especie Amenazada, y en el Apéndice II de CITES (Convención sobre el Comercio Internacional de Flora y Fauna). S. lewini se caracteriza por ser migratoria, pelágica-costera de zonas tropicales y subtropicales (Compagno, Krupp \& Schneider, 1995). Las hembras de $S$. lewini paren a sus crías en aguas costeras, ricas en nutrientes denominadas "áreas de crianza", donde los juveniles obtienen alimento y protección mientras crecen y alcanzan la madurez sexual. Al llegar a su etapa adulta el tiburón martillo realiza grandes migraciones, cambiando los hábitats costeros por zonas de aguas oceánicas y pelágicas (Clarke, 1971; Klimley, 1987; Duncan \& Holland, 2006).

Como consecuencia, sus poblaciones están siendo impactadas por distintas pesquerías: en zonas costeras los juveniles y adultos son vulnerables a las redes de enmalle (trasmallos) y líneas de fondo de pescadores artesanales, además de las redes de arrastre de embarcaciones camaroneras semi-industriales; mientras que en aguas oceánicas los adultos son capturados principalmente por líneas largas de flote o palangre pelágico (López, Arauz, Zanella \& Le Foulge, 2009).

En el ETPS se han identificado sitios de agregación de $S$. lewini en islas oceánicas, como la Isla del Coco (Costa Rica), el Archipiélago de las Galápagos (Ecuador) e Isla Malpelo (Colombia), donde se pueden observar cardúmenes de tiburones adultos de esta especie (Hearn et al., 2010; Hearn, Klimley \& Peñaherrera, 2010; Bessudo et al., 2011). Sin embargo, poco es conocido sobre el uso de hábitat de esta especie en zonas costeras (Zanella, 2008).

Para Costa Rica, diferentes autores han reportado la presencia del tiburón martillo, $S$. lewini, en capturas pesqueras comerciales en aguas costeras y pelágicas del Pacífico (Campos, 1989; Arauz, López \& Zanella, 2007; Arauz, López, Zanella, Bolaños \& Suarez, 2008; Zanella, 2008; López et al., 2009; Zanella, I.opez \& Arauz, 2009; López \& Zanella, 2011). Por ejemplo, Zanella et al. (2009) caracterizaron la pesca del S. lewini en la parte externa del Golfo de Nicoya; Campos (1989) y Fargier, Hartmann y Molina (2008) reportaron que, aunque no es una especie de importancia comercial, el tiburón martillo es capturado de manera incidental por pescadores artesanales de Golfo Dulce (La Palma, Golfito, Puerto Jiménez, Pavones, Zancudo) en faenas dirigidas a los pargos (Lutjanidae) y cabrillas (Serranidae).

Históricamente, el Golfo Dulce ha sido una fuente de recursos marino-costeros para importantes comunidades de sus alrededores como la Palma, Golfito, Puerto Jiménez, y Zancudo, entre otras comunidades. Sin embargo, la falta de investigaciones y las respectivas regulaciones en el manejo de los recursos pesqueros ha ocasionado una disminución de los mismos (Quesada \& Cortés, 2006). Por tanto, este estudio tiene como objetivo analizar los tiburones martillo capturados por pescadores artesanales en Golfo Dulce, y recomendar pautas de manejo para su conservación y uso sostenible.

\section{MATERIALES Y MÉTODOS}

Área de estudio: Golfo Dulce fue declarado Área Marina de Pesca Responsable, mediante el A.J.D.I.P. 191-2010 del INCOPESCA (La Gaceta, 2010). En este Golfo, se permite solo el uso de artes de pesca sostenibles (línea de fondo y cuerda de mano) y es prohibida de forma permanente la pesca industrial, semiindustrial y el uso de redes de arrastre.

El Golfo Dulce se ubica en el Pacífico Sur de Costa Rica, específicamente entre las coordenadas $8^{\circ} 00^{\prime}-9^{\circ} 00^{\prime} \mathrm{N}$ y $82^{\circ} 30^{\prime}-84^{\circ} 00^{\prime}$ $\mathrm{W}$ de la provincia de Puntarenas. Tiene un área de aproximadamente $700 \mathrm{~km}^{2}$ y posee características geomorfológicas muy especiales que lo convierten en uno de los sistemas de baja circulación (fiordos) descritos para zonas tropicales del mundo (Quirós, 2003).

Debido a su particular geomorfología y dinámica de aguas, se divide en dos zonas bien marcadas:

a. la zona interna, caracterizada por poseer una profunda depresión con aguas que 
llegan a más $200 \mathrm{~m}$ de profundidad y comprende el $80 \%$ del cuerpo de agua del Golfo. La circulación y recambios de agua en esta zona son restringidos, por lo que las concentraciones de oxígeno y disposición de nutrientes son bajas (Wangelin \& Wolff, 1996; Quirós, 2003).

b. la zona externa representa la boca del Golfo que se abre al Océano Pacífico, tiene solo $12 \mathrm{~km}$ de ancho y posee aguas menos profundas (hasta $60 \mathrm{~m}$ ). En esta zona se da una mejor circulación de oxígeno y una mayor productividad debido a la entrada de nutrientes ocasionada por las fuerzas de las mareas (Lei, 2002).

Recolecta de información biológica: De mayo 2010 hasta mayo 2011 en las comunidades de Puerto Jiménez y Pavones, Golfo Dulce, se recopiló información biológica-pesquera de tiburones martillo capturados durante faenas artesanales. Dicha información fue recolectada de manera separada, mediante la observación de descargas en centros de acopio y la observación a bordo de las embarcaciones pesqueras.

Para la realización de las faenas los pescadores de estas comunidades utilizan líneas de fondo, la cual consta de una línea madre de nylon con una longitud promedio de $2.1 \pm 0.8 \mathrm{~km}$, de la cual cuelgan un promedio de $308.1 \pm 90.5$ anzuelos, tipo circulares $\left(\mathrm{N}^{\circ} 10\right.$ y 12$)$ y $\mathrm{J}\left(\mathrm{N}^{\circ} 7\right.$ y 8). Para la carnada utilizan peces de escama, como sardinas (Cupleidae), jureles (Carangidae), anguilas, peces voladores (ballyhoo), entre otros. Las líneas son lanzadas en horas de la noche y madrugada, permaneciendo en el agua por periodos de 2-4 horas hasta su virado.

Durante la observación de las descargas se determinó la longitud total (LT: medida desde la punta del hocico, hasta el extremo final de la aleta caudal, $\pm 0.1 \mathrm{~cm}$ ) y el sexo (presencia o ausencia de hemipenes) de los individuos de $S$. lewini encontrados. Cuando los tiburones fueron descargados con vísceras y órganos reproductivos en su cavidad abdominal, fue posible determinar los estados reproductivos. Para esto, se utilizó una readecuación de la clasificación utilizada para el tiburón Mustelus schimitti en la Patagonia Argentina propuesta por Chiaramonte y Pettovello (2000):

\section{Machos}

Estado I: Cría o recién nacido. Hemipenes cortos y suaves, testes delgados e incoloros sin vascularización, presencia de la inserción umbilical.

Estado II: Juvenil. Hemipenes cortos y parcialmente calcificados, testículos con vascularización incipiente.

Estado III: Adulto. Hemipenes totalmente calcificados, pueden realizar una rotación de $180^{\circ}$ desde la base y volver a su posición original. Testes desarrollados, conductos deferentes en espiral color crema y vascularización visible.

Estado IV: Adulto activo (en época reproductiva). Hemipenes totalmente calcificados, testículos engrosados, vesícula seminal turgente con esperma y una alta vascularización con presencia de semen en los hemipenes.

\section{Hembras}

Estado I: Cría o recién nacida. Ovario pequeño liso e incoloro con oviductos delgados y rectos. Presencia de la inserción umbilical.

Estado II: Juvenil. Ovario con muchos folículos sin desarrollar (granulado a simple vista) y de color pálido. Útero no diferenciado del oviducto.

Estado III: Preadulta. Ovario con folículos amarillentos. Útero diferenciado y glándula oviductal en forma de corazón, útero sin óvulos.

Estado IV: Adulta. Útero largo y flácido y glándula oviductal grande en forma de corazón. Óvulos desarrollados dentro del útero.

Estado V: Grávida. Útero engrosado con embriones en su interior.

Durante los viajes de observación abordo se obtuvieron las coordenadas geográficas de la faena pesquera por medio de un sistema de Geo Posicionamiento Global (GPS), las horas exactas de pesca efectiva y el número y tipo de anzuelos calados. A los individuos de $S$. lewini encontrados se les determinó el sexo, la 
longitud total y a los machos se les determinó el estado reproductivo, utilizando la clasificación antes mencionada.

Abundancia relativa de tiburones martillo en las observaciones abordo: se calculó la abundancia relativa de $S$. lewini, utilizando la Captura Por Unidad de Esfuerzo (CPUE (Ecuación 1) a partir del número de individuos capturados por anzuelos por hora, utilizando la siguiente ecuación (López et al., 2009):

$$
\begin{gathered}
\mathrm{CPUE}=(\mathrm{TT} / \mathrm{TA})(1) \\
{[(\mathrm{HIV}-\mathrm{HIC}) / 2+(\mathrm{HFV}-\mathrm{HFC}) / 2]}
\end{gathered}
$$

donde,

$\mathrm{TT}=$ total de tiburones martillo

$\mathrm{TA}=$ total de anzuelos

$\mathrm{HIV}=$ hora inicial virado

$\mathrm{HIC}=$ hora inicial calado

$\mathrm{HFV}=$ hora final virado

$\mathrm{HFC}=$ hora final calado

La CPUE de los tiburones martillo encontrada se compararon con la CPUE estimada a partir de los datos recolectados por Zanella (2008) en la parte externa del Golfo de Nicoya. Debido a que los datos no seguían una distribución normal, la CPUE se comparó mediante la prueba estadística de Kruskall Wallis, utilizando el programa Statgraphic Centurion XVI, versión 16.1.11.

Datos biológico-pesqueros de observaciones abordo y de descargas: los datos provenientes de las descargas y de las observaciones abordo (longitud total, sexo, estados reproductivos) fueron integrados y se realizaron análisis descriptivos de los mismos.

\section{RESULTADOS}

Recolecta de información biológica: Entre mayo 2010 y mayo 2011 se recolectó información biológica-pesquera referente a 67 faenas de pescadores artesanales de Puerto Jiménez y Pavones, de estas 37 (55.2\%) fueron observaciones en centros de acopio y 30 (44.8\%) fueron abordo.
En las descargas en centros de acopio se encontraron un total de 163 tiburones martillo, mientras que durante los viajes de observación abordo se capturaron 152. En cuanto a los viajes de observación abordo, las mayores cantidades de tiburones se capturaron en las zonas de pesca conocidas como Los Bajos, Pique Fijo y Punta Banco, mientras que las menores cantidades se registraron en Corcovado, en la Piedra del Sombrero y en Matapalo (Fig. 1, Cuadro 1).

Abundancia relativa de tiburones martillo en las observaciones abordo: Los análisis de las capturas por unidad de esfuerzos (CPUE) mostraron cambios en la abundancia de tiburones martillo a lo largo del año. El bimestre con la mayor CPUE fue julio-agosto donde se capturaron 0.007 tiburones $S$. lewini por anzuelo por hora. A su vez, las menores capturas se presentaron en el bimestre mayo-junio (0.001 tiburones por anzuelo x hora) (Fig. 2).

Las CPUE calculadas a partir de las observaciones a bordo de faenas artesanales de Golfo Dulce entre mayo 2010 y mayo 2011, fueron significativamente mayores respecto a las CPUE obtenidas por el estudio de Zanella

\section{CUADRO 1}

Cantidad de tiburones martillo, Sphyrna lewini, registrados y faenas realizadas por zona de pesca. Golfo Dulce, Costa Rica, 2011

TABLE 1

Number of scalloped hammerhead sharks, Sphyrna lewini, registered and trips made by fishing area. Golfo Dulce, Costa Rica, 2011

\begin{tabular}{lcc}
\multicolumn{1}{c}{ Zona de pesca } & $\begin{array}{c}\text { Número de } \\
\text { individuos }\end{array}$ & $\begin{array}{c}\text { Número de } \\
\text { faenas observada }\end{array}$ \\
Los Bajos & 54 & 8 \\
Pique Fijo & 40 & 7 \\
Punta Banco & 26 & 1 \\
Jiménez-La Ciénaga & 23 & 6 \\
Pavones & 4 & 2 \\
Corcovado & 2 & 1 \\
Piedra del sombrero & 2 & 2 \\
Matapalo & 1 & 1 \\
Lapas Ríos & 0 & 2 \\
Total & 152 & 30 \\
\hline
\end{tabular}




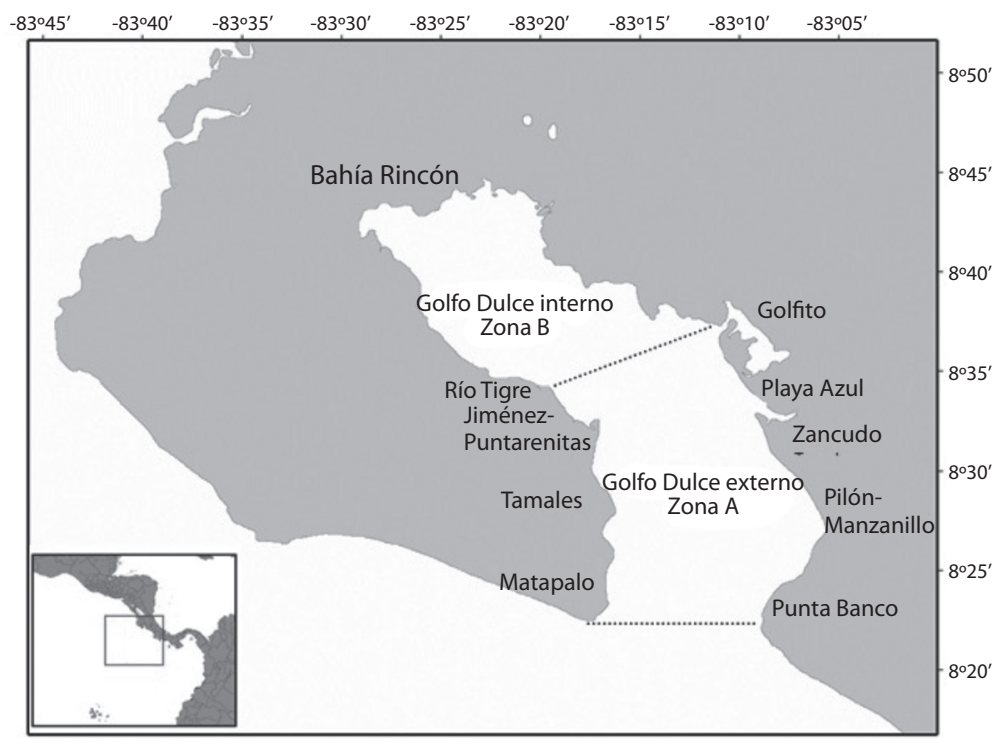

Fig. 1. Ubicación geográfica de las principales zonas pesqueras en Golfo Dulce, Costa Rica, 2011.

Fig. 1. Geographic location of fishing sites used in Golfo Dulce, Costa Rica, 2011.

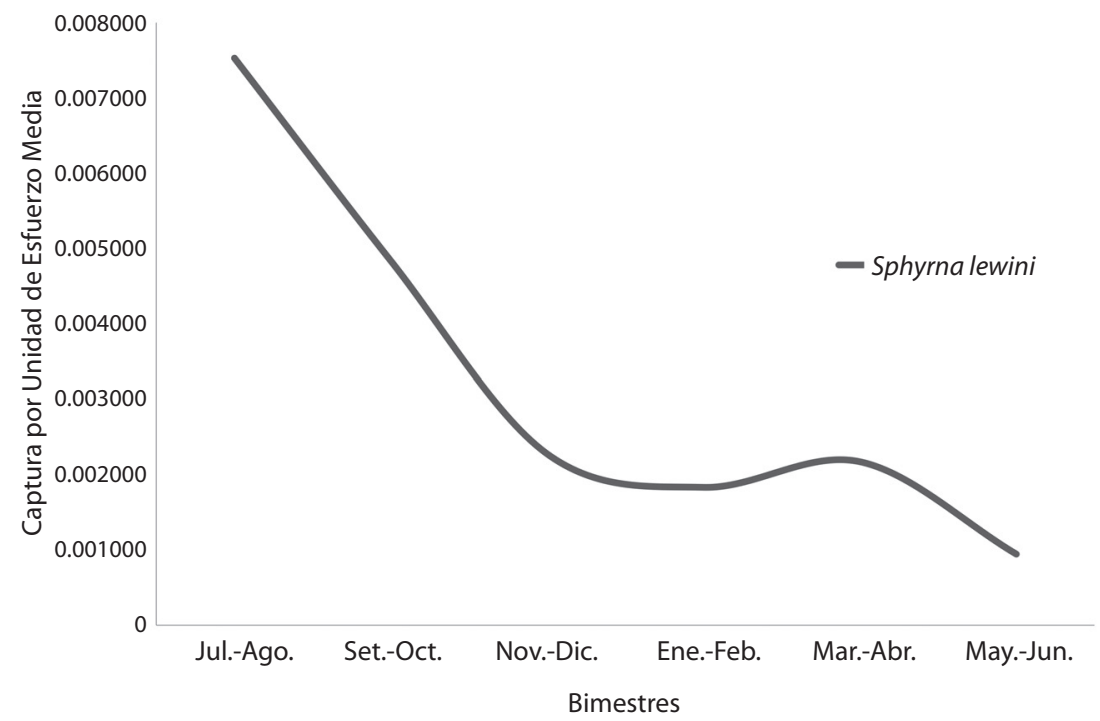

Fig. 2. Abundancia relativa anual expresada como Capturas por Unidad de Esfuerzo (CPUE) para el tiburón martillo, Sphyrna lewini, en bimestres. Golfo Dulce, Costa Rica, 2011.

Fig. 2. Annual Relative abundance for scalloped hammerhead shark, Sphyrna lewini, as Catch per Unit Effort by bimester. Golfo Dulce, Costa Rica, 2011. 
(2008) entre 2006-2007, en la zona externa del Golfo de Nicoya ( $\mathrm{H}=20.45$, $\mathrm{p}<0.0001)$ (Fig. 3).

Datos Biológico-Pesqueros de observaciones abordo y de descargas: Gracias a la integración de los datos recolectados en observaciones abordo a los recolectados en descargas, se analizaron un total de 315 individuos, de los cuales el $55.2 \%$ fueron machos y $44.8 \%$ fueron hembras, para una proporción sexual de 1.2 machos por cada hembra $\left(x^{2}=3.46\right.$; $\mathrm{p}>0.05$ ). Con respecto a los estados reproductivos, todos los individuos de $S$. lewini analizados fueron tiburones juveniles o inmaduros. De estos, el 48\% y \% 52\% presentó el Estado I y Estado II, respectivamente.

Los tiburones martillo obtuvieron una longitud total media de $74.3 \pm 17.4 \mathrm{~cm}$. La Fig. 4 muestra la distribución de frecuencia por longitud total de todos los tiburones martillos observados. La mayoría de los tiburones analizados, aproximadamente el 55\%, obtuvo longitudes totales comprendidas entre los $45 \mathrm{y}$ $75 \mathrm{~cm}$. A su vez el $40 \%$ de los tiburones martillo analizados presentaron longitudes totales comprendidas entre los 75 y $105 \mathrm{~cm}$, mientras que menos del $4 \%$ presentó longitudes entre los 120 y $150 \mathrm{~cm}$.

Se graficaron las longitudes totales medias en el tiempo y la CPUE estimada, y se observó que a lo largo de los bimestres las líneas de tendencias son inversas. En julio-agosto, cuando las longitudes totales medias fueron menores $(64 \mathrm{~cm})$, las capturas por unidad de esfuerzo fueron mayores (0.0075). Por el contrario, en enero- febrero y mayo-junio se observaron las longitudes totales medias más grandes $(86.6 \mathrm{~cm}$ y $91.8 \mathrm{~cm}$ respectivamente) y se reportaron las menores CPUE (0.0018 y 0.00019 por anzuelo por hora, respectivamente) (Fig. 5).

\section{DISCUSIÓN}

Los pescadores de Puerto Jiménez y Pavones durante sus faenas artesanales con líneas de fondo capturan al tiburón martillo en las distintas zonas de pesca ubicadas en la parte externa de Golfo Dulce. Sin embargo, más del $75 \%$ de los individuos analizados en este estudio se capturaron en las zonas conocidas como

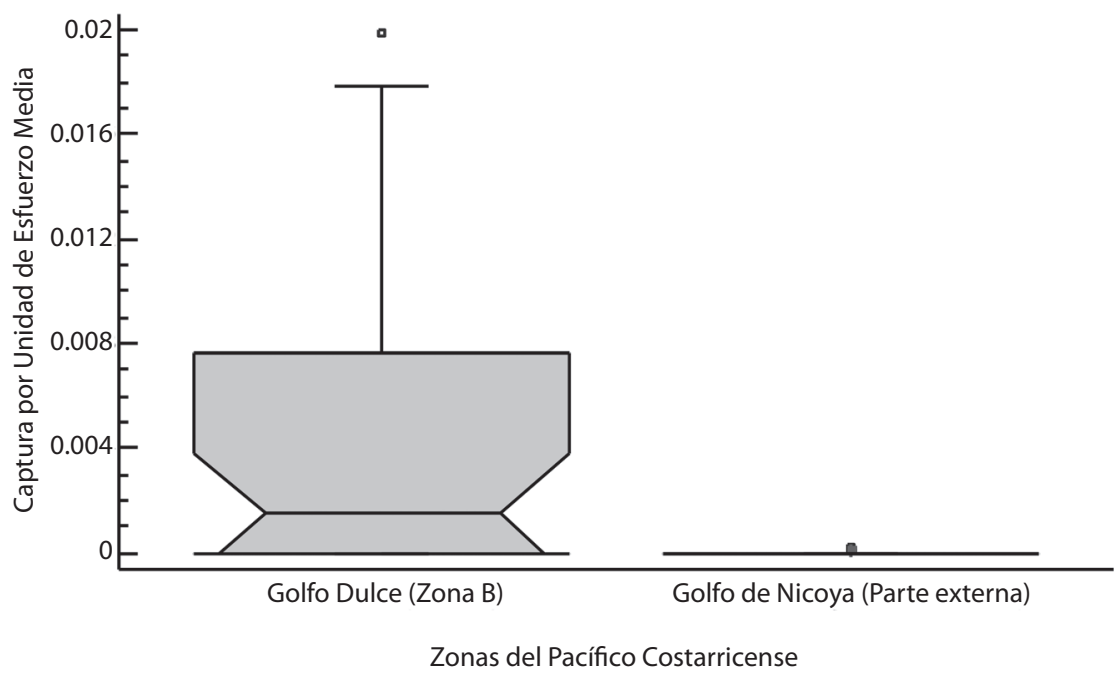

Fig. 3. Gráfico de Caja de las Capturas por Unidad de Esfuerzo (CPUE) de tiburones martillo, Sphyrna lewini, calculadas para las zonas de Golfo Dulce y Golfo de Nicoya, Costa Rica, 2011.

Fig. 3. Box plot for Catch per Unit Effort of scalloped hammerhead shark, Sphyrna lewini, estimated for Golfo Dulce and Golfo de Nicoya, Costa Rica, 2011. 


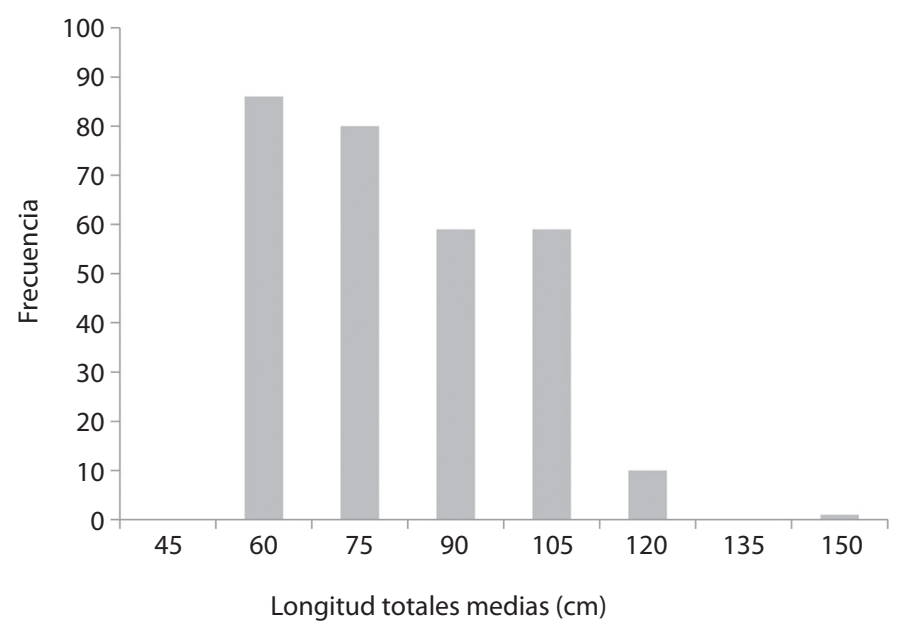

Fig. 4. Distribución de frecuencia por Longitud Total (LT) de los tiburones martillo, Sphyrna lewini, analizados abordo y en descargas. Golfo Dulce, Costa Rica 2011.

Fig. 4. Total length frequency distribution for hammerhead shark, Sphyrna lewini, analyzed during onboard and landing observations. Golfo Dulce, Costa Rica, 2011.

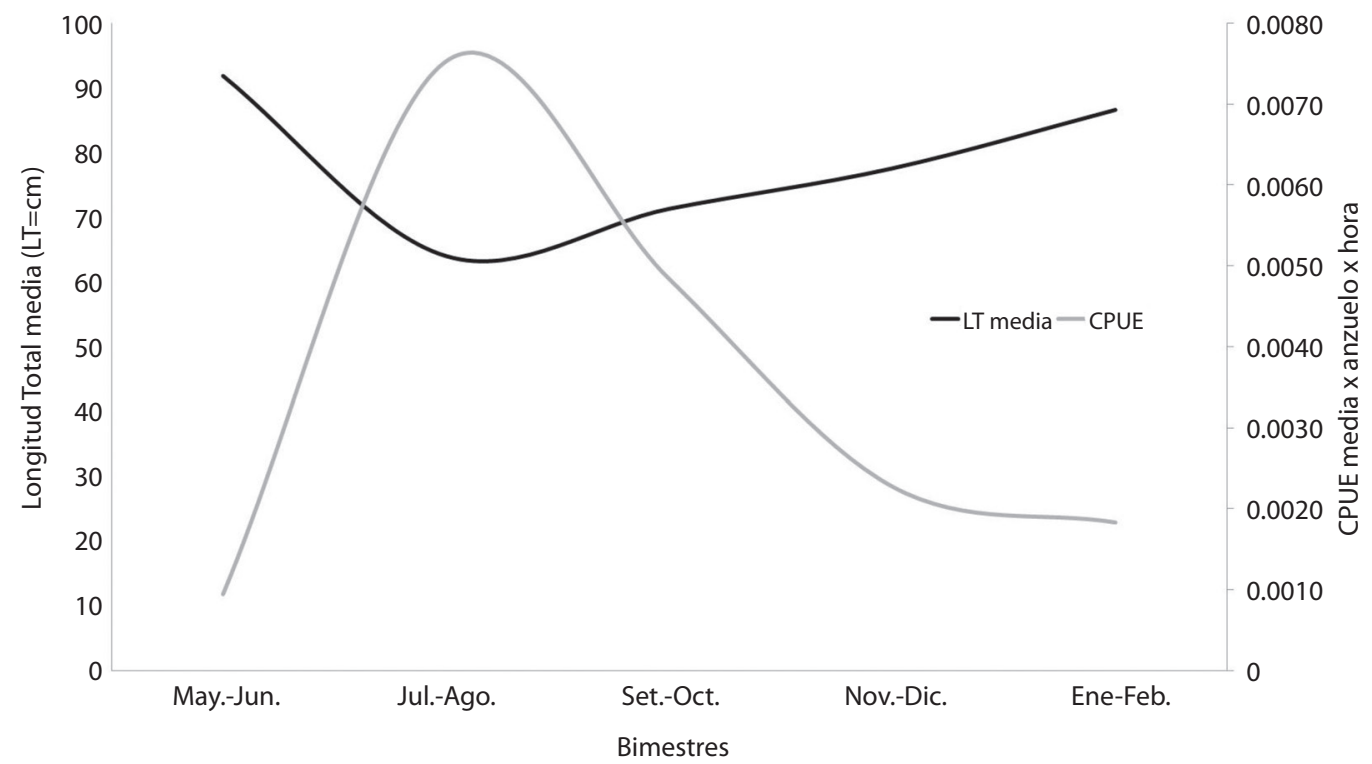

Fig. 5. Longitudes totales medias y CPUE medias por bimestres de los tiburones martillo, Sphyrna lewini, observados en Golfo Dulce, Costa Rica 2011.

Fig. 5. Mean total lengths and CPUE by bimesters of scalloped hammerhead shark, Sphyrna lewini, analyzed in Golfo Dulce, Costa Rica, 2011.

Punta Banco, La Ciénaga, Los Bajos y Pique Fijo. Debido a que el ecosistema de manglar rodea estas porciones del litoral, estas zonas se caracterizan por tener fondos lodosos-arenosos y aguas someras, turbias y ricas en nutrientes.
Las mayores capturas de $S$. lewini en estas zonas de pesca se deben a que la mayoría de las faenas se realizaron en dichos sitios, pero sobre todo, por la afinidad de S. lewini para estos tipos de hábitats. 
Distintos autores (Clarke, 1971; Castro, 1993; Simpfendorfer \& Milward, 1993; Torres, 2004; Duncan \& Holland, 2006) han reportado la preferencia de $S$. lewini (en particular de las crías y juveniles) por ecosistemas altamente productivos, relacionados a manglares y desembocaduras de ríos. Por ejemplo, Zanella et al. (2009) quienes describieron la pesca de $S$. lewini en la zona externa del Golfo de Nicoya, Costa Rica, reportaron una fuerte relación entre juveniles de S. lewini y la zona conocida como el Peñón, ubicada cerca de la desembocadura del Río Tárcoles.

De la misma forma, en el presente estudio las longitudes totales de los tiburones martillo (entre los 46 y $140 \mathrm{~cm}$ ) y el análisis de los estados reproductivos confirmaron que los individuos de S. lewini encontrados fueron crías y juveniles. De hecho, todos los tiburones analizados presentaron longitudes inferiores a la talla de primera madurez sexual reportada por Branstetter (1987) y Anislado (2001). El primer autor determinó que en el Golfo de México las hembras maduran a los $250 \mathrm{~cm}$ con 15 años de edad y los machos a los $180 \mathrm{~cm}$ con 10 años de edad. Mientras que, según Anislado (2001) en el litoral de Michoacán de México las hembras maduran a los $190 \mathrm{~cm}$ con 4.4 años de edad y los machos a los $170 \mathrm{~cm}$ con 3.6 años.

La proporción sexual encontrada para la especie concuerda con los resultados de otros autores, tales como Aguilar (2003) y Zanella (2008), quienes reportaron una proporción sexual para $S$. lewini de 1.5 y 1.18 machos por cada hembra en el Golfo de California (México) y de Nicoya (Costa Rica), respectivamente. Lo anterior posiblemente se deba a una estrategia de segregación sexual de la especie. De hecho, Klimley (1987), en el Bajo Espíritu Santo, Baja California, México, describió una segregación sexual entre machos y hembras de $S$. lewini; según el autor, estas últimas crecen más rápido y se desplazan hacia aguas más profundas.

Las observaciones abordo de las embarcaciones pesqueras fueron muy valiosas para estimar la abundancia relativa de $S$. lewini a lo largo del año. Al igual que lo reportado por Duncan y Holland (2006), que caracterizaron la población de $S$. lewini en Bahía Kane’ohe, Hawaii, los juveniles del tiburón martillo se capturaron a lo largo de todo el año. Sin embargo, las abundancias relativas más altas se reportaron durante meses de la época lluviosa, en particular en el bimestre julio-agosto. Esta época implica una alta descarga de nutrientes proveniente de los ríos, ofreciendo a los tiburones aguas aún más productivas y turbias, brindándoles alimento y protección durante las primeras etapas de su ciclo de vida (Zanella et al., 2009). Sin embargo, al comparar las abundancias relativas (CPUE) y las longitudes totales medias de los tiburones martillo por bimestre, se observan tendencias opuestas. En el bimestre de julio-agosto cuando las longitudes medias fueron las menores, las CPUE fueron mayores. Esto sugiere que durante los meses de julio y agosto hay un aumento en las abundancias de las crías del tiburón martillo.

En el Área Marina Protegida del Santuario de Flora y Fauna de Malpelo, Bessudo et al. (2011) reportan que la gran mayoría de los tiburones martillo encontrados en los decomisos de pesca ilegal corresponden a hembras grávidas. Además, los autores concluyen que entre marzo y abril los tiburones martillo dejan Malpelo, probablemente para dirigirse hacia aguas costeras a dar a luz sus crías. De la misma forma, Hearn et al. (2010) en los islotes Darwin y Wolf, en el Archipiélago de Galápagos (Ecuador) encontraron que la abundancia de tiburones martillo disminuye entre marzo y junio. Por tanto, debido a que los tiburones martillo son especies vivíparas con ciclos anuales y su copulación, gestación y alumbramiento son sincronizados y relacionados con la abundancia relativa de las presas (Hamlett, 1999), se puede concluir que las hembras grávidas posiblemente lleguen a Golfo Dulce para parir a sus crías durante los primeros meses de la época lluviosa (mayo y junio), alcanzando un pico en la abundancia relativa de las crías en julio y agosto. De esta forma, el periodo de alumbramiento coincidiría con los patrones de migración reportados por los investigadores en Malpelo y Galápagos. Además, esta conclusión concuerda con lo reportado 
por Poirout (2008), que analizó las faenas de pesca de pescadores artesanales de Zancudo en Golfo Dulce, y encontró una hembra grávida de $S$. lewini en mayo.

También Anislado (2001) en la costa de Michoacana, México, reporta la época de nacimientos de crías de $S$. lewini entre mayo y julio. Según el autor, esta estrategia reproductiva de parir las crías durante los meses iniciales del invierno, aumenta la supervivencia de los juveniles del tiburón martillo que aprovechan el aumento en nutrientes y presas en aguas costeras consecuencia de las lluvias. A su vez, Bejarano (2007), quién describió la biología reproductiva de $S$. lewini en Salina Cruz, Oaxaca, México, determinó que el nacimiento de las crías del tiburón martillo ocurre en julio y agosto.

Gracias a la comparación de CPUE entre las obtenidas en Golfo Dulce y las calculadas para la parte externa del Golfo de Nicoya, se sugiere que en Golfo Dulce alberga una importante población de tiburones martillo para el litoral Pacífico de Costa Rica y posiblemente para la región del ETPS. De hecho Golfo Dulce, podría ser una importante área de crianza para S. lewini. Sin embargo, según Kinney \& Simpfendorfer (2009) para confirmar la existencia de las áreas de crianza se necesita demostrar lo siguiente: 1) que hay una mayor abundancia de tiburones en el área comparada con otras, 2) que los tiburones tienen una tendencia a permanecer en el área por largos periodos, y 3) que es utilizada por distintas generaciones a lo largo de los años. De tal forma, que solo el estudio a largo plazo podrá confirmar si Golfo Dulce es realmente un área de crianza para el tiburón martillo, lo cual lo convertiría en un importante hábitat crítico para la especie en la región del ETPS.

Finalmente, para promover la sostenibilidad de $S$. lewini en el ETPS, se recomienda implementar vedas temporales durante el periodo de nacimiento de las crías de tiburón martillo (junio-agosto) en sitios donde se reportaron las mayores capturas: Punta Banco, Pique Fijo, Los Bajos y La Ciénaga. Es necesario implementar las vedas, ya que la liberación de individuos en esta especie no representa una efectiva estrategia de manejo: López y Zanella (2011) reportan una sobrevivencia de aproximadamente un $15 \%$ en tiburones martillo capturados con líneas de fondo artesanal en Golfo Dulce.

Además, es imprescindible establecer un estudio a largo plazo que permita el monitoreo de los sitios donde se reportan las mayores capturas de $S$. lewini; esto con el fin de evaluar si representan un área de crianza para la especie. Asimismo, es necesario dar seguimiento a sus capturas y evaluar la factibilidad de las acciones de conservación recomendadas.

\section{AGRADECIMIENTOS}

Este estudio fue posible solo por el apoyo de diferentes pescadores artesanales de Golfo Dulce, en especial de Puerto Jiménez y Pavones. Gracias a José Collado, Pedro Rodríguez, Pedrito Rodríguez, William Mata, por su cooperación y por permitirnos las capturas de tiburones martillo de sus faenas pesqueras. Gracias por el apoyo financiero a Conservación Internacional Centroamérica, Columbus and Aquarium Zoo, Mohammed Bin Zayed Conservation Fund y Marine Action Conservation Fund. Se agradece el Proyecto Consolidación de Áreas Marinas Protegidas del Sistema Nacional de Áreas de Conservación, Programa de Naciones Unidas para el Desarrollo y el Global Environment Facility (GEF) por su colaboración en la publicación.

\section{RESUMEN}

En Golfo Dulce, Costa Rica, se han reportado capturas incidentales de $S$. lewini por pescadores artesanales. Debido a esto, este estudio tiene como objetivo caracterizar las capturas del tiburón martillo en Golfo Dulce, y recomendar pautas de manejo. Entre mayo 2010 y mayo 2011 se recopiló información biológica-pesquera de tiburones capturados durante faenas artesanales en Golfo Dulce. Se estimó la captura por unidad de esfuerzo a lo largo del año. Se analizaron un total de 315 tiburones, todos juveniles, con una longitud total media de $74.3 \pm 17.4 \mathrm{~cm}$. Las longitudes totales y la abundancia relativa tuvieron tendencias opuestas: en julio-agosto cuando las longitudes totales medias fueron menores, las capturas por unidad de esfuerzo 
fueron mayores. Debido a esto, se recomienda implementar vedas temporales durante el periodo de nacimiento de las crías de tiburón martillo (junio-agosto) en sitios donde se reportaron las mayores capturas.

Palabras clave: Tiburón martillo, Sphyrna lewini, área de crianza, pesca artesanal, Golfo Dulce.

\section{REFERENCIAS}

Abercrombie, D. L., Clarke, S., \& Mahmood, S. (2005). Global-scale genetic identification of hammerhead sharks: Application to assessment of the international fin trade and law enforcement. Conservation Genetics, 6, 775-788.

Aguilar, A. (2003). Ecología trófica de juveniles del tiburón martillo Sphyrna lewini (Griffith \& Smith, 1834) en el Golfo de California (Tesis de Maestría). Instituto Politécnico Nacional Centro Interdisciplinario de Ciencias marinas (CICIMAR), La Paz, México.

Anislado, T., \& Mendoza, C. B. (2001). Edad y crecimiento del tiburón martillo Sphyrna lewini (Griffith y Smith 1834) en el Pacífico Central de México. Ciencias Marinas, 27, 501-520.

Arauz, R., López A., \& Zanella I. (2007). Análisis de la descarga anual de tiburones y rayas en la pesquería pelágica y costera del Pacífico de Costa Rica (Playas del Coco, Tárcoles y Golfito) (Informe final). San José, Costa Rica.

Arauz R., López, A., Zanella, I., Bolaños, A., \& Suarez, R. (2008). Análisis de las capturas y descargas de tiburones en las pesquerías del Pacífico de Costa Rica (Playas del Coco y Golfito). San José, Costa Rica: Conservación Internacional Centroamérica.

Barker, M. J., \& Schluessel, V. (2005). Managing global shark fisheries: suggestions for prioritizing management strategies. Aquatic Conservation, 15, 325-347.

Baum, J. K., Myers, R., Kehler, D., Worm, B., Harley, S., \& Doherty, P. (2003). Collapse and conservation of shark populations in the Northwest Atlantic. Science, 299, 389-392.

Bejarano, M. (2007). Biología reproductiva del tiburón martillo Sphyrna lewini (Griffith y Smith, 1834) en Salina Cruz, Oaxaca, México (Tesis de Maestría). Instituto Politécnico Nacional Centro Interdisciplinario de Ciencias marinas (CICIMAR), La Paz, Baja California, México.

Bessudo, S., Soler, G., Klimley, P., Ketchum, J., Hearn, A., \& Arauz, R. (2011). Residency of the scalloped hammerhead shark (Sphyrna lewini) at Malpelo Island and evidence of migration to other islands in the Eastern Tropical Pacific. Environmental Biology of Fishes, 91, 165-176.
Branstetter, S. (1987). Age, growth and reproductive biology of the silky shark, Carcharhinus falciformis, and the scalloped hammerhead, Sphyrna lewini, from thenorthwestern Gulf of Mexico. Environmental Biology of Fishes, 19, 161-173.

Campos, J. (1989). Evaluación de la pesca artesanal del Golfo Dulce (Proyecto cooperativo Ministerio de Agricultura y Ganadería, Universidad de Costa Rica). San José, Costa Rica: CIMAR, Universidad de Costa Rica.

Castro, J. (1993). The Nursery of Bull Bay, South Carolina, with a Review of the Shark Nurseries of the Southeastern Coast of the United States. Environmental Biology of Fishes, 38, 37-48.

Chiaramonte, G., \& Pettovello, P. (2000). The biology of Mustelus schimitti in southern Patagonia, Argentina. Journal of Fish Biology, 57, 930-942.

Clarke, T. A. (1971). The ecology of the scalloped hammerhead shark, Sphyrna lewini, in Hawaii. Pacific Science, 25, 133-144.

Compagno, L., Krupp, F., \& Schneider, W. (1995). Tiburones. In W. Fischer, F. Krupp, W. Schneider, C. Sommer, K. Carpenter, \& V. Niem (Eds.), Guía para la identificación de especies para los fines de pesca (pp. 647-744). Roma, Italia: Pacífico Centro-Oriental.

Duncan, K., \& Holland, K. (2006). Habitat use, growth rates and dispersal patterns of juvenile scalloped hammerhead sharks Sphyrna lewini in a nursery habitat. Marine Ecology Progress Series, 312, 211-221.

Fargier, L., Hartmann, H., \& Molina, H. (2008). Desarrollo de un proceso de monitoreo participativo en las comunidades pesqueras de Bahía Pavones (Golfo Dulce) en el Pacífico Sur de Costa Rica (Informe). San José, Costa Rica.

Hamlett, W. C. (1999). Sharks, skates and rays: the biology of elasmobrach fishes. Baltimore, Maryland. USA: The John Hopkins University Press.

Hearn A., Utreras, E., \& Henderson S. (Eds.) (2010). Informe sobre el estado de los tiburones del Pacífico Este Tropical. Quito, Ecuador: Conservación Internacional.

Hearn A., Klimley, P., \& Peñaherrera, C. (2010). Hotspots within hotspots? Hammerhead shark movements around Wolf Island. Galapagos Marine Reserve. Marine Biology, 157, 1899-1915.

Kinney, M. J., \& Simpfendorfer, C. A. (2009). Reassessing the value of nursery areas to shark conservation and management. Conservation Letters, 2, 53-60.

Kitchell, J. F., Essington, T., Boggs, C., Schindler, D., \& Walters, C. (2002). The Role of Sharks and Longline Fisheries in a Pelagic Ecosystem of the Central Pacific. Ecosystems, 5, 202-216. 
Klimley, P. (1987). The determinants of sexual segregation in the scalloped hammerhead shark, Sphyrna lewini. Environmental Biology of Fishes, 18, 27-40.

La Gaceta $N^{\circ}$ 159. (2010). Diario Oficial Costa Rica. Decreto INCOPESCA AJDPI/251. San José, Costa Rica.

Lei, Z. (2002). Marine coastal dynamics and primary production response in Golfo Dulce, Costa Rica: A multi-sensor satellite approach (Master's thesis). International Institute for Geoinformation Science and Earth Observation, Enschede, Holanda.

López, A., Arauz R., Zanella, I., \& Le Foulge, L. (2009). Análisis anual de las capturas de tiburones y rayas en las pesquerías artesanales de Tárcoles, Pacífico Central de Costa Rica. Revista Ciencias Marina y Costera, 1, 145-157.

López, A., \& Zanella, I. (2011). Conservación del tiburón martillo (Sphyrna lewini) en Golfo Dulce (Informe para Conservación Internacional). San José, Costa Rica.

Myers, R. A., Baum, J. K., Shepherd, T. D., Powers, S. P., \& Peterson, C. H. (2007). Cascading effects of the loss of apex predatory sharks from a coastal ocean. Science, 315, 1846-1850.

Poirout, T. (2008). Diagnóstico socio ambiental de la pesca artesanal por una gestión participativa de la zona costera de zancudo (Golfo Dulce, Costa Rica) (Tesis de Maestría). Université de La Rochelle, France.

Quesada, M., \& Cortés, J. (2006). Capítulo XII. Golfo Dulce. In V. Nielsen \& M. Quesada (Eds.), Informe técnico: Ambientes marino costeros de Costa Rica. San José, Costa Rica: CIMAR, Conservation International, TNC.

Quirós, G. (2003). Circulación del Golfo Dulce: un fiordo tropical.Tópicos Meteorológicos y Oceanográficos, 10, 75-83.
Rowe, S., \& Hutchings, J. (2003). Mating system and the conservation of commercially exploited marine fishes. Department of Biology, Dalhouise University. Trends in Ecology and Evolution, 18, 567-572.

Simpfendorfer, C. A., \& Milward, N. E. (1993). Utilization of a tropical bay as a nursery area by sharks of the families Carcharhinidae and Sphyrnidae. Environmental Biology of Fishes, 37, 337-345.

Távarez, R. (2005). Abundance and distribution of sharks in Los Roques Archipelago National Park and other Venezuelan oceanic islands 1997-1998. Ciencias Marinas, 31, 441-454.

Torres, H. A. (2004). Distribución, abundancia y hábitos alimentarios de juveniles del tiburón martillo Sphyrna lewini Griffith y Smith (SPHYRNIDAE) en la costa de Sinaloa, México durante el evento El Niño 1997-98 (Tesis de maestría). Universidad deñ Mar, Oaxaca, México.

Walker, T. (1998). Can shark resources be harvested sustainably? A question revisited with a review of shark fisheries. Marine Freshwater Research, 49, 553-572.

Wangelin, M., \& Wolff, M. (1996). Comparative biomass spectra and species composition of the zooplankton communities in Golfo Dulce and Golfo de Nicoya, Pacific coast of Costa Rica. Revista Biología Tropical, 44, 133-155.

Zanella, I. (2008). Caracterización de la pesca del tiburón martillo (Sphyrna lewini) y algunos aspectos sobre distribución, reproducción y alimentación en el Pacifico central de Costa Rica. (Tesis de Maestría). Universidad Nacional, Heredia, Costa Rica.

Zanella, I., Lopez, A., \& Arauz, R. (2009). Caracterización de las descargas del tiburón martillo, Sphyrna lewini, en un área de crianza ubicada en el Golfo de Nicoya, Costa Rica. Revista de Ciencias Marinas y Costeras, 1,175-195. 
